\title{
Characterization of the Stratified Morphology of Nanoparticle Agglomerates
}

Andrea Fabre $^{a}$, Teun Steur ${ }^{a}$, Wim G. Bouwman ${ }^{b}$, Michiel T. Kreutzer ${ }^{a}$, J. Ruud van Ommen ${ }^{a, *}$

${ }^{a}$ Delft University of Technology, Department of Chemical Engineering, Van der Maasweg 9, 2629 HZ Delft, the Netherlands

${ }^{b}$ Delft University of Technology, Department of Radiation, Science and Technology, Mekelweg 15, 2629 JB Delft, The Netherlands 


\section{Supporting Information}

\section{Values obtained from SESANS}

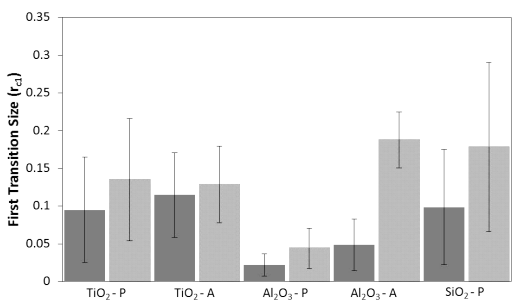

(a)

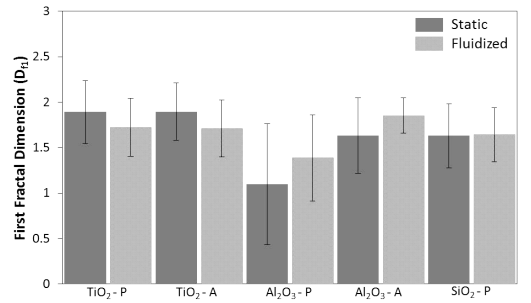

(b)

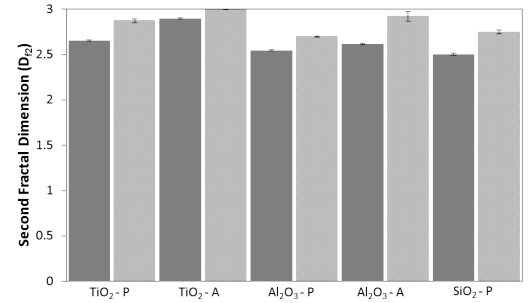

(c)

Figure 1: Plots of the transition size between the first and second scales estimated from SESANS, and the fractal dimension of the first and second scales estimated when left as fitting parameters.

Evaluation of the second prefactor and transition size between simple and complex agglomerates. At the intersection points, the size and density of both scales are the same. Also, in order to meet the boundary condition of a single nanoparticle, $\hat{k_{n 1}}$ must be equal to 1 . To estimate the second prefactor we used:

$$
\begin{gathered}
\hat{k_{n 1}}\left(\frac{d_{a 1}}{d_{p}}\right)^{D_{f 1}-3}=\hat{k_{n 2}}\left(\frac{d_{a 2}}{d_{p}}\right)^{D_{f 2}-3} \\
d_{a 1}=d_{a 2}=r_{c 1} \\
\hat{k_{n 1}}=1 \\
\hat{k_{n 2}}=\left(\frac{r_{c 1}}{d_{p}}\right)^{D_{f 1}-D_{f 2}}
\end{gathered}
$$

For the transition size between the simple and complex agglomerates we got:

$$
d_{a 2}=d_{a 3} \longrightarrow d_{c 2}
$$




$$
\begin{gathered}
\hat{k_{n 2}}\left(\frac{d_{c 2}}{d_{p}}\right)^{D_{f 2}-3}=\hat{k_{n 3}}\left(\frac{d_{c 2}}{d_{p}}\right)^{D_{f 3}-3} \\
d_{c 2}=d_{p}\left(\frac{\hat{k_{n 2}}}{\hat{k_{n 3}}}\right)^{\frac{1}{D_{f 3}-D_{f 2}}}
\end{gathered}
$$

